\title{
Inverse design of nanophotonic devices with structural integrity
}

\author{
Yannick Augenstein*, ${ }^{* \dagger}$ and Carsten Rockstuhl*,,$+ \dagger$ \\ $\dagger$ Institute of Theoretical Solid State Physics, Karlsruhe Institute of Technology, 76131 \\ Karlsruhe, Germany \\ $\ddagger$ Institute of Nanotechnology, Karlsruhe Institute of Technology, 76021 Karlsruhe, Germany \\ E-mail: yannick.augenstein@kit.edu; carsten.rockstuhl@kit.edu
}

\begin{abstract}
Computational inverse design has been a driving force behind the development of compact and highly efficient nanophotonic devices. However, due to fabrication constraints, devices have so far mostly been restricted to planar geometries. With recent developments, additive manufacturing techniques are poised to open up a vast design space for free-form nanophotonic devices, bringing with them a new set of inverse design challenges. The most urgent one is structural integrity. With a technique such as 3D laser nanolithography (nearly) every structure can be written, but not every structure is self supported and is with that feasible; free-floating elements are simply not an option. To address this challenge, we present here a method for the inverse design of nanophotonic devices that combines electromagnetic and structural topology optimization. To illustrate the proposed method, we present designs for a nanolens and a mode converter with structural integrity. We show that some of these designs achieve efficiencies comparable to those of conventional nanophotonic inverse design while maintaining structural integrity; and even slightly surpass them. This opens up
\end{abstract}


new possibilities for photonic device design and may lead to the development of novel photonic devices for additive manufacturing.

\section{Keywords}

Inverse design, Topology optimization, Additive manufacturing, Photonics, Compliance minimization

\section{Introduction}

Computational methods for the inverse design of novel nanophotonic devices and artificial photonic materials with predefined functionalities have received significant interest in recent years $^{115}$ and have led to the development of highly efficient designs across a range of applications. ${ }^{[6]}$ Large-scale, gradient-based optimization of device geometries, i.e. topology optimization, has been enabled by the adjoint method, $\stackrel{15] 16}{ }$ which permits the calculation of gradients with respect to an arbitrary number of degrees of freedom with only two full-field simulations. In a sense, however, this design freedom has not been utilized to its full potential, with topology optimization being primarily used to design planar devices, $\frac{17-21}{21}$ with

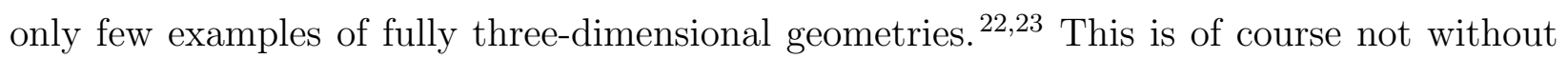
good reasons, and chiefly among these are restrictions imposed by available manufacturing techniques. However, recent advancements in additive manufacturing techniques such as 3D laser nanolithography $\sqrt[24 \mid 25]{25}$ have put fabrication of fully three-dimensional micro-structures well within reach. In particular, these technologies promise to offer optical devices with functionalities on demand, if and only if the necessary blueprints for these designs are available. With manufacturing catching up, it is time to think about topology-optimized free-form geometries for nanophotonics and the new challenges that this brings to inverse design.

The central problem that we tackle in this work is the requirement that free-form structures written by additive manufacturing techniques, such as 3D laser nanolithography, need 
to consist of fully connected material layouts, as any element without structural support simply collapses. Structural integrity is a property that, to the authors' knowledge, has not been considered in electromagnetic topology optimization so far. We hypothesize that this is due to the fact that development in topology optimization for photonics has largely focused on planar devices that can be produced by traditional subtractive manufacturing. For such devices, structural integrity is of no concern since structural support is always given by the surface that they are etched into. However, no such assumption can be made for free-form geometries. Here, we present a new paradigm for topology optimization in nanophotonics in which we aim to solve this problem by introducing compliance minimization into the optimization problem. We first outline the method for solving both structural as well as electromagnetic topology optimization problems simultaneously. Afterwards, we use the developed method to design a photonic nanolens and a waveguide-integrated mode converter that feature an increasing degree of structural integrity. Our method can be applied more generally to design devices that combine both optical and mechanical properties, opening up a new class of functional elements for nanophotonics.

\section{Methods}

Topology-optimized devices in nanophotonics naturally tend towards wavelength-scale features. While this generally leads to highly efficient devices, it is detrimental to structural integrity. For the inverse design of devices with structural integrity, we turn to a different discipline in which topology optimization has long been established: structural mechanics. $\frac{26}{28}$ In mechanical topology optimization, the nature of the problem dictates that the resulting devices are mechanically stable, and this necessitates the existence of linked structures in the solution. Our method makes use of this property by combining electromagnetic and mechanical topology optimization into a single framework that enables the simultaneous optimization of both structural and optical objectives. By specifying external forces that act 
on the structure, the device's structural integrity with regards to these loads is considered during optimization. The direction along which material connectivity is established depends on the distribution of these forces. They can be applied in a purely fictitious manner, e.g. if the material of the device should be connected along a specific axis one can artificially introduce forces on both ends of the design that act parallel to this axis. Likewise, the forces can represent physical loads acting on the structure. The latter approach is preferable if the structural loads can be estimated in advance. We have included examples for both cases in this work.

Because our method relies on the integration of both structural topology optimization in the form of compliance minimization as well as electromagnetic topology optimization, we will briefly outline both approaches independently in this section and then proceed to define the coupled optimization problem.

\section{Topology optimization for nanophotonics and structural mechanics}

For both the structural and electromagnetic topology optimization we use a density-based material parametrization, $\frac{29[30}{20}$ where the material is represented by a vector $\boldsymbol{\rho}$ of continuous design variables $\rho_{i} \in[0,1]$ that are mapped to the physical material distribution by means of a series of differentiable transformations for each pixel in the simulation domain. To achieve sufficiently large and binary features in the optimized designs, filtering and projection schemes $^{31 / 32}$ are applied during the optimization and the final design density $\hat{\tilde{\rho}}$ is obtained.

We first apply a Gaussian filter to the design variables to avoid small features in the optimized design,

$$
\tilde{\rho}_{i}=\frac{\sum_{j \in \mathcal{D}_{i}} w_{i j} \rho_{j}}{\sum_{j \in \mathcal{D}_{i}} w_{i j}} \quad \text { with } \quad w_{i j}= \begin{cases}r_{\min } \exp \left(-\frac{\left|\boldsymbol{r}_{i}-\boldsymbol{r}_{j}\right|^{2}}{2 \sigma^{2}}\right) & \forall \boldsymbol{r}_{j} \in \mathcal{D}_{i} \\ 0 & \text { otherwise }\end{cases}
$$

where $\mathcal{D}_{i}$ is the set of elements that lie within the filter radius $r_{\min }$ of element $i$ and $w_{i j}$ are 
the respective weights of the filtering kernel. The standard deviation is given by $\sigma=r_{\min } / \sqrt{3}$, which has a similar behavior as the commonly used cone filter, $\stackrel{33}{,}$ where we choose a minimum feature size of $r_{\min }=100 \mathrm{~nm}$. A soft threshold (i.e. projection filter) given by

$$
\hat{\tilde{\rho}}_{i}=\frac{\tanh (\alpha \beta)+\tanh \left(\alpha\left(\tilde{\rho}_{i}-\beta\right)\right)}{\tanh (\alpha \beta)+\tanh (\alpha(1-\beta))}
$$

is applied to the filtered density to promote binary solutions in the optimization, where $\alpha$ denotes the steepness of the curve and $\beta$ its center. For the optimizations presented in this work, values of $\alpha=30$ and $\beta=0.5$ were used.

After filtering and projection, the design density is linearly interpolated to yield the permittivities

$$
\boldsymbol{\epsilon}_{r}=\epsilon_{\min }+\hat{\tilde{\boldsymbol{\rho}}}\left(\epsilon_{\max }-\epsilon_{\min }\right)
$$

for the electromagnetic simulation and Young's modulus

$$
\boldsymbol{Y}=Y_{\min }+\hat{\tilde{\boldsymbol{\rho}}}\left(Y_{\max }-Y_{\min }\right)
$$

for the structural analysis, respectively. We note that, with the exception of the final material interpolation, the parametrization in both simulations is identical. Otherwise, one design density would lead to two distinct material distributions, causing the structural and electromagnetic simulations to consider different geometries.

For the electromagnetic optimization, we simulate the optical response from the structure using the finite-difference frequency-domain (FDFD) method. $\stackrel{34}{34}$ The optimization is formulated as the minimization of an electromagnetic objective $F_{\mathrm{EM}}(\boldsymbol{\rho})$ with respect to the design 
variables $\rho$ :

$$
\begin{array}{ll}
\min _{\boldsymbol{\rho}} & F_{\mathrm{EM}}(\boldsymbol{\rho}) \\
\text { s.t. } & \boldsymbol{\nabla} \times \frac{1}{\mu_{0}} \boldsymbol{\nabla} \times \boldsymbol{E}-\omega^{2} \mu_{0} \epsilon_{0} \boldsymbol{\epsilon}_{r}(\boldsymbol{\rho}) \boldsymbol{E}=-i \omega \boldsymbol{j} \\
& \mathbf{0} \leq \boldsymbol{\rho} \leq \mathbf{1},
\end{array}
$$

with the electric field $\boldsymbol{E}$, the electromagnetic current source $\boldsymbol{j}$ and relative permittivity $\boldsymbol{\epsilon}_{r}$. We have assumed a relative permeability of $\boldsymbol{\mu}_{r}=1$ everywhere. The electromagnetic objective $F_{\mathrm{EM}}(\boldsymbol{\rho})$ is defined depending on the optimization problem and its gradients with respect to the design variables are obtained by adjoint sensitivity analysis. $\frac{16135}{.135}$

The structural optimization is formulated as a compliance minimization problem and solved using an in-house implementation of the direct stiffness method:36

$$
\begin{array}{ll}
\min _{\boldsymbol{\rho}} & F_{\mathrm{C}}(\boldsymbol{\rho})=\boldsymbol{U}^{\top} \mathbf{K} \boldsymbol{U}=\sum_{e=1}^{N} Y_{e}\left(\rho_{e}\right) \boldsymbol{u}_{e}^{\top} \mathbf{k}_{0} \boldsymbol{u}_{e} \\
\text { s.t. } & \mathbf{K} \boldsymbol{U}=\boldsymbol{F} \\
& \mathbf{0} \leq \boldsymbol{\rho} \leq \mathbf{1}
\end{array}
$$

where the objective $F_{\mathrm{C}}(\boldsymbol{\rho})$ is the compliance, $\mathbf{K}$ is the global stiffness matrix, $\boldsymbol{U}$ is the global displacement vector, $\mathbf{k}_{0}$ is the unit element stiffness matrix, $\boldsymbol{u}_{e}$ is the element displacement vector, and $\boldsymbol{F}$ is the vector of mechanical forces applied to the system. The stiffness of each of the $N$ elements is given by the design-variable dependent Young's modulus $Y_{e}\left(\rho_{e}\right)$. Typically, an additional volume constraint $\int_{\Omega} \boldsymbol{\rho} \mathrm{d} \Omega \leq V$ is imposed on the optimization such that the material volume in the optimization domain $\Omega$ never exceeds a specified volume fraction $V$. However, we do not impose such a constraint as this would unnecessarily restrict the design space for the optical optimization and the electromagnetic design goal naturally prevents a trivial solution. Additionally, volume constraints are generally not an issue in the design of nanophotonic devices. For topology optimization, the sensitivity of the compliance 
with respect to the design variables needs to be known and is given as:

$$
\frac{\partial F_{\mathrm{C}}}{\partial \rho_{e}}=-\frac{\partial \hat{\tilde{\rho}}_{e}}{\partial \rho_{e}}\left(Y_{\max }-Y_{\min }\right) \boldsymbol{u}_{e}^{\top} \mathbf{k}_{0} \boldsymbol{u}_{e}
$$

where $\partial \hat{\tilde{\rho}}_{e} / \partial \rho_{e}$ depends on the chosen filtering and projection scheme.

\section{The optimization problem}

By choosing the same discretization for both the structural and electromagnetic problem, a single set of design variables can be used to construct the material geometry for both simulations and we can combine both the compliance and the optical objectives into a single objective function and formulate a coupled optimization problem:

$$
\begin{array}{ll}
\min _{\boldsymbol{\rho}} & F(\boldsymbol{\rho})=\left(1-\omega_{\mathrm{C}}\right) F_{\mathrm{EM}}(\boldsymbol{\rho})-\omega_{\mathrm{C}} F_{\mathrm{C}}(\boldsymbol{\rho})+F_{\mathrm{B}}(\boldsymbol{\rho}) \\
\text { s.t. } & \boldsymbol{\nabla} \times \frac{1}{\mu_{0}} \boldsymbol{\nabla} \times \boldsymbol{E}-\omega^{2} \mu_{0} \epsilon_{0} \boldsymbol{\epsilon}_{r}(\boldsymbol{\rho}) \boldsymbol{E}=-i \omega \boldsymbol{j} \\
& \mathbf{K}(\boldsymbol{\rho}) \boldsymbol{U}=\boldsymbol{F} \\
& \mathbf{0} \leq \boldsymbol{\rho} \leq \mathbf{1},
\end{array}
$$

where we introduce the compliance factor $\omega_{\mathrm{C}} \in[0,1]$ to prioritize the terms in the objective function. High compliance factors give a higher weight to the structural term, which should lead to more connected structures at the expense of the electromagnetic figure of merit.

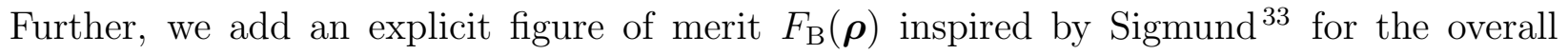
binarization of the structure:

$$
F_{\mathrm{B}}(\boldsymbol{\rho})=\min \left(-\log \left(\frac{\sum_{i=1}^{n} 4 \hat{\tilde{\rho}}_{i}\left(1-\hat{\tilde{\rho}}_{i}\right)}{n}\right), \gamma\right)
$$

This term is only added once the optimization is close to convergence, i.e. the relative change

in the value of the total figure of merit falls below a certain threshold (here $1 \times 10^{-3}$ ). The 
parameter $\gamma$ limits the maximum value of the binarization objective. In the examples given in this work we use a value of $\gamma=2$, which has been found to generally lead to good binarization while remaining numerically stable.

The gradients of each term in the objective function as well as those of the material parametrization are calculated using automatic differentiation. $\frac{3738}{3}$ This approach allows to change both the objective function and material parametrization without the need to derive new gradients by hand with each modification.

While the resulting material distributions tend towards connected features, they represent a compromise between the mechanical and optical optimization and thus do not entirely guarantee the absence of any free-floating elements. This is solved by using a multi-step optimization procedure. First, the optimization is run once until convergence. Any freefloating elements are then removed from the material distribution during a post-processing step by connected component labelling. ${ }^{39}$ The processed material distribution is then further refined during a second optimization run, yielding final designs consisting of only connected material.

\section{Example applications}

We demonstrate the outlined approach by applying it to the inverse design of two different nanophotonic devices. For each device, we perform multiple optimizations by sweeping the value of the compliance factor $\omega_{\mathrm{C}}$ across the range $[0,1]$, where a value of 0 corresponds to regular electromagnetic topology optimization (our baseline) and a value of 1 completely disregards the optical design objective. The driving forces of the structural simulation are scaled such that the lower bound of the compliance is in the same order of magnitude as the final optical figure of merit $F_{\mathrm{EM}}(\boldsymbol{\rho})$ of the baseline optimization at $\omega_{\mathrm{C}}=0$. In all examples we choose a minimum element stiffness of $Y_{\min }=1 \times 10^{-6}$ and a maximum element stiffness of $Y_{\max }=1$ for the structural analysis. For the electromagnetic simulations we choose 
an operating wavelength of $\lambda=1 \mu \mathrm{m}$. Once binarized, the media can be made from a medium with a refractive index of $n=1.5$, as a typical value for polymers used in 3D laser nanolithography, or air for which we assumed a refractive index of $n=1$. The simulation domain has an area of $12 \mu \mathrm{m} \times 8 \mu \mathrm{m}$ at a resolution of $30 \mathrm{~mm}^{-1}$ and perfectly matched layers with a thickness of $d_{\mathrm{PML}}=1 \mu \mathrm{m}$ on each side. For the sake of simplicity, we limit ourselves to $2 \mathrm{D}$ simulations in this work, but note that all equations in the previous section are valid also in $3 \mathrm{D}$.

Our focus in this work lies on the inverse design of functional photonic elements with the added benefit of structural integrity. Thus, the sources of the electromagnetic simulation are known as they are imposed by the problem statement for each device. However, the definition of the loads for the structural problem is oftentimes less obvious. Mechanical forces are generally not a concern for nanophotonic devices, and the authors are not aware of any load-bearing optical elements. Our goal is much rather the generation of devices with connected features that can be produced by additive manufacturing techniques, and such connected designs are the only class of feasible solutions in compliance problems. This means that in many applications, the physical forces acting on the device play only a minor role. In such cases, we can introduce fictitious forces acting on the device that guide the optimization towards structures that are connected along a certain axis. However, we stress that our approach can accommodate arbitrary load cases and can be used without modification for physical loads.

The simulations are implemented in Python and we use the L-BFGS algorithm $\underline{40}$ as implemented in the open-source library nlopt $\underline{41}$ for local gradient-based optimization. Each optimization takes roughly 30 minutes on a personal computer (Intel Core i7-7700) to converge. 


\section{Photonic nanolens}

In the first example, we apply our optimization approach to the design of a photonic nanolens. The setup of the problem is illustrated in Fig. 1. The device consists of two areas of solid

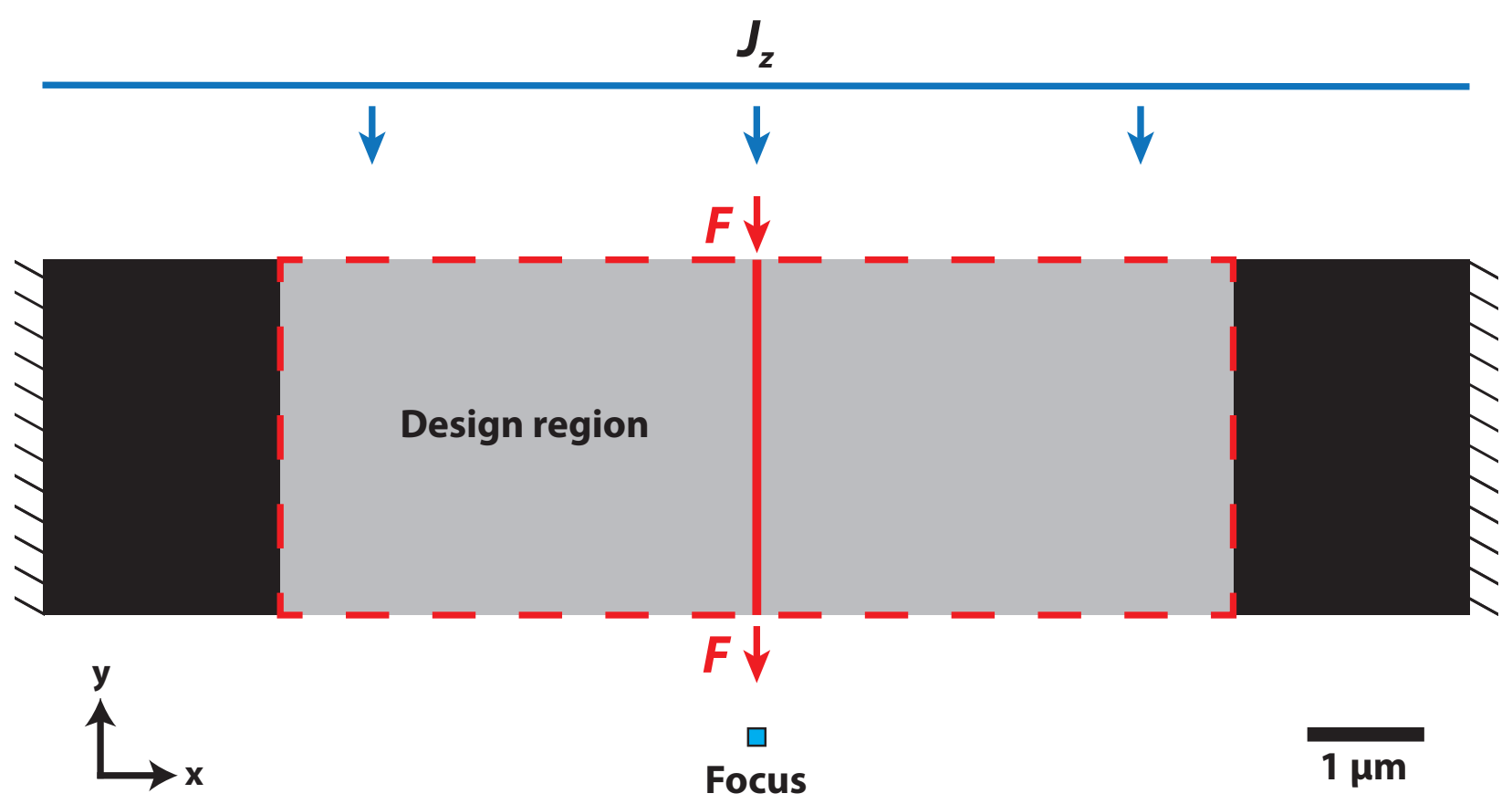

Figure 1: Design setup for the optimization of a nanophotonic focussing element. The design region (dashed red rectangle) has an area of $8 \mu \mathrm{m} \times 3 \mu \mathrm{m}$ and lies in between two solid slabs of material $(n=1.5)$ that are fixed on either end of the simulation domain and surrounded by air $(n=1)$. An $E_{z}$-polarized (out-of-plane) plane wave source with an operating wavelength of $1 \mu \mathrm{m}$ placed at the top of the simulation illuminates the structure. The electromagnetic design goal is to increase the intensity of the electric field in the focal spot located $1.5 \mathrm{um}$ below the lower edge of the structure. The structural problem is defined as the compliance minimization of the material $\left(Y_{\max }=1\right)$ within the design region with respect to a vertical load applied along the center of the design.

material on either side of the domain that are separated by a design area which will contain the optimized material distribution. The structure is illuminated by a plane wave that is linearly polarized normal to the plane shown and impinges on the top of the design at normal incidence. The optical design goal is to focus this light into a small focal spot below the design region. We define the electromagnetic figure of merit $F_{\mathrm{EM}}$ as the intensity of the 
electric field $E_{z}$ in the focal region $\mathcal{M}$ :

$$
F_{\mathrm{EM}}=\frac{\int_{\mathcal{M}}\left|E_{z}\right|^{2} \mathrm{~d} A}{\int_{\mathcal{D}}\left|E_{z}\right|^{2} \mathrm{~d} A}
$$

where we divide by the intensity of the field in the design region $\mathcal{D}$ to avoid designs that exploit strong field enhancement due to resonances inside the design region, as such designs are extremely susceptible to fabrication imperfections. Maximizing the electric field intensity in a small region of space, i.e. in a small square with an area of $60 \mathrm{~nm} \times 60 \mathrm{~nm}$, has been shown to lead to tight focal spots in inverse design problems. ${ }^{42}$

We assume that the structure is mechanically held in place on the left and right edges of the domain. This could be realized by placing e.g. some structural support pillars to which these blocks are connected outside of the simulation domain. A vertical load is placed along the center of the design region representing the weight of the lens element. The goal of the structural optimization is then to maximize the stiffness of the device with respect to such a vertical load, which can only be achieved if the device is also anchored to the fixed material at the left and right sides of the design region. While this example is presented as a toy problem, it should be noted that in the rotationally symmetric case, the problem setup resembles that of free-form fiber coupling elements ${ }^{43 / 44}$ or microlens systems. $\cdot \frac{45546}{}$

The baseline design at $\omega_{\mathrm{C}}=0$ depicted in Fig. 2(a) exhibits multiple disjunct elements in the design domain. While the purely electromagnetic topology optimization came up with a lens design with a tight focal spot, the resulting device clearly cannot be manufactured by 3D laser nanolithography. However, the optimized designs Fig. 2(b)-(d) at higher compliance factors offer well-connected structures with increasing stiffness. None of the designs contain free-floating elements, while achieving a qualitatively similar field profile as the base design. At higher compliance factors, the relative weight of the compliance term in the optimization becomes dominant and we can see a degradation of the intensity in the focal region.

In Fig. 2(e) we have plotted the electromagnetic figure of merit $F_{\mathrm{EM}}(\boldsymbol{\rho})$ and the corre- 

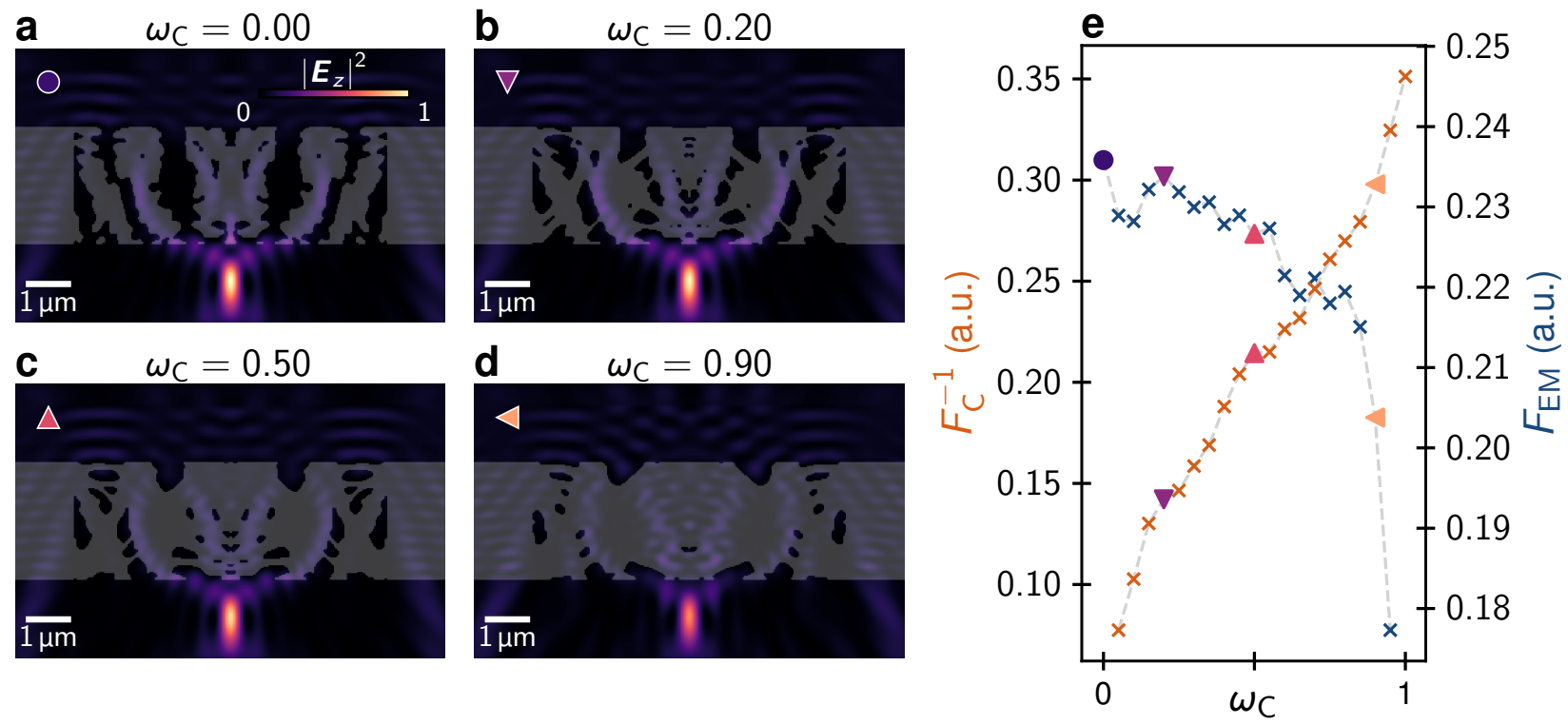

Figure 2: Optimization results for the photonic nanolens. a-d Optimized designs overlaid on top of their respective field intensities $\left|E_{z}\right|^{2}$ (normalized)for compliance factors $\omega_{\mathrm{C}}$ of $0.0,0.2,0.5$ and 0.9 . e The electromagnetic figure of merit $F_{\mathrm{EM}}$ (blue) and the material stiffness $F_{\mathrm{C}}$ (orange) for optimized lens designs at evenly spaced compliance factors between 0 and 1 . The designs in $\mathbf{a}-\mathbf{d}$ are indicated by markers. The values of $F_{\mathrm{C}}$ at $\omega_{\mathrm{C}}=0$ and $F_{\mathrm{EM}}$ at $\omega_{\mathrm{C}}=1$ are not shown since optimizations are purely optical or mechanical at these compliance factors. 
sponding material stiffness $F_{\mathrm{C}}^{-1}(\boldsymbol{\rho})$ for the designs. We see that increasing the compliance factor generally comes at the expense of the optical design goal. However, the design at $\omega_{\mathrm{C}}=0.2$ achieves a field intensity that is only $0.8 \%$ smaller than that of the base design while consisting only of a single connected structure. All together it can be seen at this example, that structurally stable and well-performing photonic nanolenses can be achieved with this design method.

\section{Mode converter}

Mode converters are a prototypical $\stackrel{47}{4}$ example for integrated photonic devices commonly

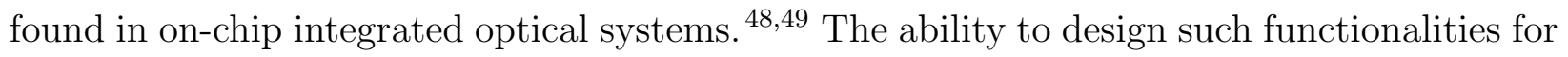
free-form geometries could potentially lead to integration of these designs into e.g. photonic wire bonds. 50151 Such photonic wire bonds are free from waveguide written by 3D laser nanolithography that connect different optical chips. A necessary requirement for this is being able to come up with device designs that do not have free-floating elements. In this second example, we design a mode converter that converts from the fundamental to the second order TE mode in a waveguide as illustrated in the optimization setup in Fig. 3.

We excite the fundamental TE mode of the waveguide on the left side of the design and define the objective as the overlap of the input electric field $\boldsymbol{E}$ with the output mode field $\boldsymbol{E}_{\mathrm{TE} 2}$ over the surface $S$ of a field monitor $\mathcal{M}$ placed at the right hand side of the simulation:

$$
F_{\mathrm{EM}}=\left|\int_{\mathcal{M}} \boldsymbol{E}_{\mathrm{TE} 2}^{*} \boldsymbol{E} \mathrm{d} S\right|
$$

In this part of the work the optimization is done with the FDFD method while the final evaluation of the coupling efficiency has been done with the finite-difference time-domain (FDTD) method. $\frac{52}{52}$

The baseline design in Fig. 4(a) achieves a mode conversion efficiency of $98.6 \%$. In the material distribution there are several disjunct elements, the most prominent of which is a 


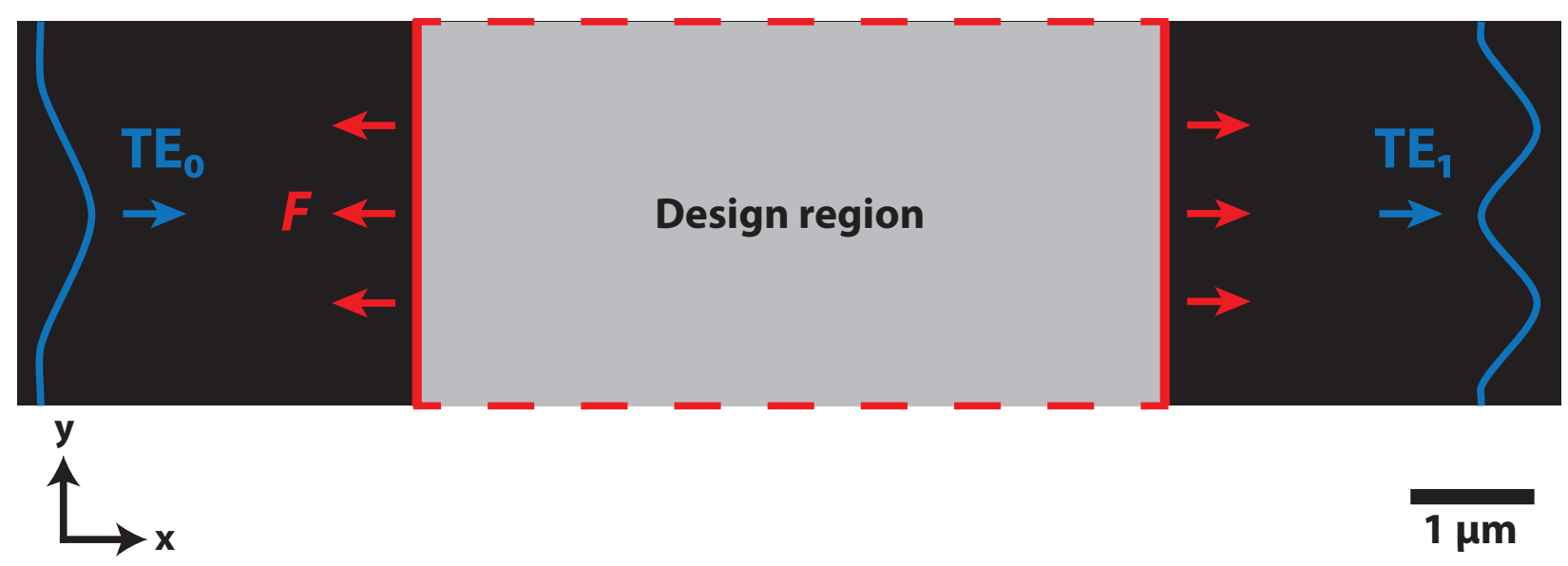

Figure 3: Design setup for the optimization of a waveguide mode converter. The design region (dashed red rectangle) has an area of $4 \mu \mathrm{m} \times 6 \mu \mathrm{m}$ and is embedded in a waveguide $(n=1.5)$ with a width of $3 \mu \mathrm{m}$ surrounded by air $(n=1)$. The fundamental $\mathrm{TE}_{0}$ mode with an operating wavelength of $1 \mu \mathrm{m}$ is injected into the waveguide from the left. The electromagnetic design goal is the maximization of the mode overlap with the second order $\mathrm{TE}_{1}$ mode at the right end of the waveguide. The structural problem is defined as the compliance minimization of the material $\left(Y_{\max }=1\right)$ within the design region with respect to two opposing forces placed at its left and right edges.
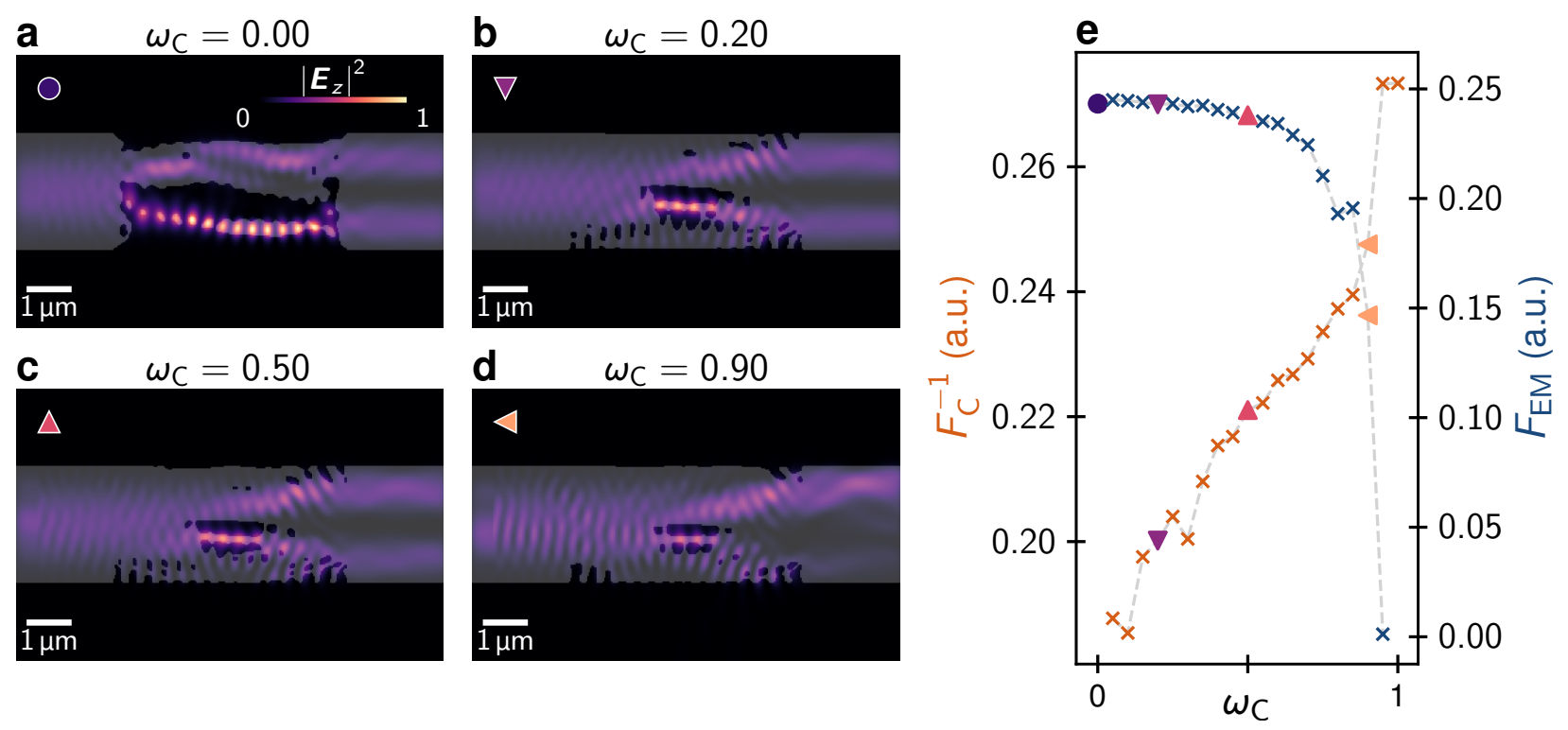

Figure 4: Optimization results for the mode converter. a-d Optimized designs overlaid on top of their respective field intensities $\left|E_{z}\right|^{2}$ (normalized) for compliance factors $\omega_{\mathrm{C}}$ of $0.0,0.2,0.5$ and 0.9 . e The electromagnetic figure of merit $F_{\mathrm{EM}}$ (blue) and the material stiffness $F_{\mathrm{C}}$ (orange) for optimized lens designs at evenly spaced compliance factors between 0 and 1 . The designs in $\mathbf{a}-\mathbf{d}$ are indicated by markers. The values of $F_{\mathrm{C}}$ at $\omega_{\mathrm{C}}=0$ and $F_{\mathrm{EM}}$ at $\omega_{\mathrm{C}}=1$ are not shown since optimizations are purely optical or mechanical at these compliance factors. 
long ridge-like structure guiding the field in the lower half of the design, making it infeasible for integration into a free-form waveguide. We can again observe that by increasing the compliance factor, the optimization finds designs with growing structural integrity (Fig. 4(b)(d)) at the cost of their coupling efficiencies for large $\omega_{\mathrm{C}}$, plotted in Fig. 4(e). However, we observe that the coupling efficiencies for designs up to compliance factors of 0.3 are as high as or even higher than that of the base design, with the largest coupling efficiency being $99.2 \%$ at $\omega_{\mathrm{C}}=0.1$. Essentially, this means that there is a free lunch - up to this compliance factor, increasing structural integrity does not come at the cost of decreasing its optical functionality! A mode converter consisting of only connected elements such as the one depicted in Fig. 4(b) could potentially be integrated into free-form geometries and realized by additive manufacturing techniques.

\section{Discussion}

In this work, we have developed a topology optimization scheme that incorporates both optical and mechanical design objectives. We demonstrated that the method is capable of designing well-connected devices that are suitable for additive manufacturing by introducing mechanical loads during the optimization. Importantly, we observe that the inclusion of mechanical objectives does not necessarily lead to an immediate degradation in the optical figure of merit and can in certain cases even aid in finding better solutions than purely electromagnetic topology optimization.

For simplicity, we have only considered two-dimensional geometries in this work, but an extension to three dimensions is straightforward. We have also not included methods to increase robustness towards manufacturing imperfections such as the commonly used

erosion-dilation scheme, $\frac{5354}{5 u t}$ these techniques can be integrated in our method without modification.

In summary, we have extended electromagnetic topology optimization by combining it 
with structural analysis to simultaneously optimize both optical as well as structural objectives of functional photonic devices. Moreover, functional devices that depend on both their mechanical and optical properties can now be tackled using topology optimization. A possible application could be better designs for retinal cell scaffolds ${ }^{55}$ that optimally distribute light to the lower sensory layers. Our work is a step towards large-scale optimization of fully free-form device geometries in nanophotonics that are suited for additive manufacturing.

\section{Acknowledgement}

This research has been funded by the Deutsche Forschungsgemeinschaft (DFG, German Research Foundation) under Germany's Excellence Strategy via the Excellence Cluster 3D Matter Made to Order (EXC-2082/1, Grant No. 390761711) and via the SFB 1173 (Grant No. 258734477). Y.A. acknowledges support from the Carl Zeiss Foundation via the CZFFocus@HEiKA program.

\section{References}

(1) Molesky, S.; Lin, Z.; Piggott, A. Y.; Jin, W.; Vucković, J.; Rodriguez, A. W. Inverse Design in Nanophotonics. Nat. Photonics 2018, 12, 659-670.

(2) Kadic, M.; Milton, G. W.; van Hecke, M.; Wegener, M. 3D Metamaterials. Nat. Rev. Phys. 2019, 1, 198-210.

(3) Li, W.; Meng, F.; Chen, Y.; fan Li, Y.; Huang, X. Topology Optimization of Photonic and Phononic Crystals and Metamaterials: A Review. Adv. Theory Simul. 2019, 2, 1900017.

(4) Schneider, P.-I.; Garcia Santiago, X.; Soltwisch, V.; Hammerschmidt, M.; Burger, S.; Rockstuhl, C. Benchmarking Five Global Optimization Approaches for Nano-Optical 
Shape Optimization and Parameter Reconstruction. ACS Photonics 2019, 6, 27262733.

(5) Wiecha, P. R.; Muskens, O. L. Deep Learning Meets Nanophotonics: A Generalized Accurate Predictor for Near Fields and Far Fields of Arbitrary 3D Nanostructures. Nano Lett. 2020, 20, 329-338.

(6) Piggott, A. Y.; Petykiewicz, J.; Su, L.; Vučković, J. Fabrication-Constrained Nanophotonic Inverse Design. Sci. Rep. 2017, 7, 1-7.

(7) Wang, J.; Shi, Y.; Hughes, T.; Zhao, Z.; Fan, S. Adjoint-Based Optimization of Active Nanophotonic Devices. Opt. Express 2018, 26, 3236-3248.

(8) Hughes, T. W.; Minkov, M.; Williamson, I. A. D.; Fan, S. Adjoint Method and Inverse Design for Nonlinear Nanophotonic Devices. ACS Photonics 2018, 5, 4781-4787.

(9) Pestourie, R.; Pérez-Arancibia, C.; Lin, Z.; Shin, W.; Capasso, F.; Johnson, S. G. Inverse Design of Large-Area Metasurfaces. Opt. Express 2018, 26, 33732-33747.

(10) Lin, Z.; Groever, B.; Capasso, F.; Rodriguez, A. W.; Lončar, M. Topology-Optimized Multilayered Metaoptics. Phys. Rev. Appl. 2018, 9, 044030.

(11) Su, L.; Piggott, A. Y.; Sapra, N. V.; Petykiewicz, J.; Vučković, J. Inverse Design and Demonstration of a Compact On-Chip Narrowband Three-Channel Wavelength Demultiplexer. ACS Photonics 2018, 5, 301-305.

(12) Lin, Z.; Liu, V.; Pestourie, R.; Johnson, S. G. Topology Optimization of Freeform Large-Area Metasurfaces. Opt. Express 2019, 27, 15765-15775.

(13) Wang, E. W.; Sell, D.; Phan, T.; Fan, J. A. Robust Design of Topology-Optimized Metasurfaces. Opt. Mater. Express 2019, 9, 469-482. 
(14) Kudyshev, Z. A.; Kildishev, A. V.; Shalaev, V. M.; Boltasseva, A. Machine-LearningAssisted Topology Optimization for Highly Efficient Thermal Emitter Design. Conference on Lasers and Electro-Optics (2019), Paper FTh3C.2. 2019; p FTh3C.2.

(15) Giles, M. B.; Pierce, N. A. An Introduction to the Adjoint Approach to Design. Flow, Turbul. Combust. 2000, 65, 393-415.

(16) Lalau-Keraly, C. M.; Bhargava, S.; Miller, O. D.; Yablonovitch, E. Adjoint Shape Optimization Applied to Electromagnetic Design. Opt. Express 2013, 21, 21693-21701.

(17) Jensen, J. S.; Sigmund, O. Topology Optimization of Photonic Crystal Structures: A High-Bandwidth Low-Loss T-Junction Waveguide. J. Opt. Soc. Am. B 2005, 22, 11911198.

(18) Sell, D.; Yang, J.; Doshay, S.; Yang, R.; Fan, J. A. Large-Angle, Multifunctional Metagratings Based on Freeform Multimode Geometries. Nano Lett. 2017, 17, 3752-3757.

(19) Hughes, T.; Veronis, G.; Wootton, K. P.; England, R. J.; Fan, S. Method for Computationally Efficient Design of Dielectric Laser Accelerator Structures. Opt. Express 2017, 25, 15414-15427.

(20) Dory, C.; Vercruysse, D.; Yang, K. Y.; Sapra, N. V.; Rugar, A. E.; Sun, S.; Lukin, D. M.; Piggott, A. Y.; Zhang, J. L.; Radulaski, M.; Lagoudakis, K. G.; Su, L.; Vučković, J. Inverse-Designed Diamond Photonics. Nat. Commun. 2019, 10, 1-7.

(21) Xie, Z.; Lei, T.; Qiu, H.; Zhang, Z.; Wang, H.; Yuan, X. Broadband On-Chip Photonic Spin Hall Element via Inverse Design. Photonics Res. 2020, 8, 121-126.

(22) Deng, Y.; Korvink, J. G. Topology Optimization for Three-Dimensional Electromagnetic Waves Using an Edge Element-Based Finite-Element Method. Proc. R. Soc. A 2016, 472, 20150835. 
(23) Camayd-Muñoz, P.; Ballew, C.; Roberts, G.; Faraon, A. Multifunctional Volumetric Meta-Optics for Color and Polarization Image Sensors. Optica 2020, 7, 280-283.

(24) Barner-Kowollik, C.; Bastmeyer, M.; Blasco, E.; Delaittre, G.; Müller, P.; Richter, B.; Wegener, M. 3D Laser Micro- and Nanoprinting: Challenges for Chemistry. Angew. Chem., Int. Ed. 2017, 56, 15828-15845.

(25) Hahn, V.; Kiefer, P.; Frenzel, T.; Qu, J.; Blasco, E.; Barner-Kowollik, C.; Wegener, M. Rapid Assembly of Small Materials Building Blocks (Voxels) into Large Functional 3D Metamaterials. Adv. Funct. Mater. 30, 1907795.

(26) Bendsoe, M. P. Optimization of Structural Topology, Shape, and Material; SpringerVerlag: Berlin Heidelberg, 1995.

(27) Rozvany, G. Aims, Scope, Methods, History and Unified Terminology of ComputerAided Topology Optimization in Structural Mechanics. Struct. Multidisc. Optim. 2001, 21, 90-108.

(28) Bendsoe, M. P.; Sigmund, O. Topology Optimization: Theory, Methods, and Applications, 2nd ed.; Springer-Verlag: Berlin Heidelberg, 2004.

(29) Bendsøe, M. P. Optimal Shape Design as a Material Distribution Problem. Struct. Optim. 1989, 1, 193-202.

(30) Sigmund, O.; Maute, K. Topology Optimization Approaches: A Comparative Review. Struct. Multidisc. Optim. 2013, 48, 1031-1055.

(31) Wang, F.; Lazarov, B. S.; Sigmund, O. On Projection Methods, Convergence and Robust Formulations in Topology Optimization. Struct. Multidisc. Optim. 2011, 43, 767784.

(32) Zhou, M.; Lazarov, B. S.; Wang, F.; Sigmund, O. Minimum Length Scale in Topology 
Optimization by Geometric Constraints. Compu. Methods Appl. Mech. Eng. 2015, 293, 266-282.

(33) Sigmund, O. Morphology-Based Black and White Filters for Topology Optimization. Struct. Multidisc. Optim. 2007, 33, 401-424.

(34) fancompute/fdfdpy. Fan Group, 2020.

(35) Jensen, J. S.; Sigmund, O. Topology Optimization for Nano-Photonics. Laser Photonics Rev. 2011, 5, 308-321.

(36) Sigmund, O. A 99 Line Topology Optimization Code Written in Matlab. Struct. Multidisc. Optim. 2001, 21, 120-127.

(37) HIPS/autograd. Harvard Intelligent Probabilistic Systems Group, 2020.

(38) Hughes, T. W.; Williamson, I. A. D.; Minkov, M.; Fan, S. Forward-Mode Differentiation of Maxwell's Equations. ACS Photonics 2019, 6, 3010-3016.

(39) Virtanen, P. et al. SciPy 1.0: Fundamental Algorithms for Scientific Computing in Python. Nat. Methods 2020, 17, 261-272.

(40) Liu, D. C.; Nocedal, J. On the Limited Memory BFGS Method for Large Scale Optimization. Math. Program. 1989, 45, 503-528.

(41) Johnson, S. G. stevengj/nlopt. 2020.

(42) Augenstein, Y.; Vetter, A.; Lahijani, B. V.; Herzig, H. P.; Rockstuhl, C.; Kim, M.S. Inverse Photonic Design of Functional Elements That Focus Bloch Surface Waves. Light: Sci. Appl. 2018, 7, 1-9.

(43) Dietrich, P.-I.; Blaicher, M.; Reuter, I.; Billah, M.; Hoose, T.; Hofmann, A.; Caer, C.; Dangel, R.; Offrein, B.; Troppenz, U.; Moehrle, M.; Freude, W.; Koos, C. In Situ 3D 
Nanoprinting of Free-Form Coupling Elements for Hybrid Photonic Integration. Nat. Photonics 2018, 12, 241-247.

(44) Gissibl, T.; Thiele, S.; Herkommer, A.; Giessen, H. Sub-Micrometre Accurate FreeForm Optics by Three-Dimensional Printing on Single-Mode Fibres. Nat. Commun. 2016, 7, 1-9.

(45) Gissibl, T.; Thiele, S.; Herkommer, A.; Giessen, H. Two-Photon Direct Laser Writing of Ultracompact Multi-Lens Objectives. Nat. Photonics 2016, 10, 554-560.

(46) Thiele, S.; Arzenbacher, K.; Gissibl, T.; Giessen, H.; Herkommer, A. M. 3D-Printed Eagle Eye: Compound Microlens System for Foveated Imaging. Sci. Adv. 2017, 3, e1602655.

(47) Miller, D. A. B. All Linear Optical Devices Are Mode Converters. Opt. Express 2012, 20, 23985-23993.

(48) Frellsen, L. F.; Ding, Y.; Sigmund, O.; Frandsen, L. H. Topology Optimized Mode Multiplexing in Silicon-on-Insulator Photonic Wire Waveguides. Opt. Express 2016, 24, 16866-16873.

(49) Piggott, A. Y.; Ma, E. Y.; Su, L.; Ahn, G. H.; Sapra, N. V.; Vercruysse, D.; Netherton, A. M.; Khope, A. S. P.; Bowers, J. E.; Vučković, J. Inverse-Designed Photonics for Semiconductor Foundries. ACS Photonics 2020, 7, 569-575.

(50) Lindenmann, N.; Balthasar, G.; Hillerkuss, D.; Schmogrow, R.; Jordan, M.; Leuthold, J.; Freude, W.; Koos, C. Photonic Wire Bonding: A Novel Concept for Chip-Scale Interconnects. Opt. Express 2012, 20, 17667-17677.

(51) Lindenmann, N.; Dottermusch, S.; Goedecke, M. L.; Hoose, T.; Billah, M. R.; Onanuga, T. P.; Hofmann, A.; Freude, W.; Koos, C. Connecting Silicon Photonic 
Circuits to Multicore Fibers by Photonic Wire Bonding. J. Lightwave Technol. 2015, 33, $755-760$.

(52) Oskooi, A. F.; Roundy, D.; Ibanescu, M.; Bermel, P.; Joannopoulos, J. D.; Johnson, S. G. Meep: A Flexible Free-Software Package for Electromagnetic Simulations by the FDTD Method. Comput. Phys. Commun. 2010, 181, 687-702.

(53) Sigmund, O. Manufacturing Tolerant Topology Optimization. Acta Mech. Sin. 2009, 25, 227-239.

(54) Liu, J.; Ma, Y. A Survey of Manufacturing Oriented Topology Optimization Methods. Adv. Eng. Softw. 2016, 100, 161-175.

(55) Hippler, M.; Lemma, E. D.; Bertels, S.; Blasco, E.; Barner-Kowollik, C.; Wegener, M.; Bastmeyer, M. 3D Scaffolds to Study Basic Cell Biology. Adv. Mater. 2019, 31, 1808110. 


\section{Graphical TOC Entry}

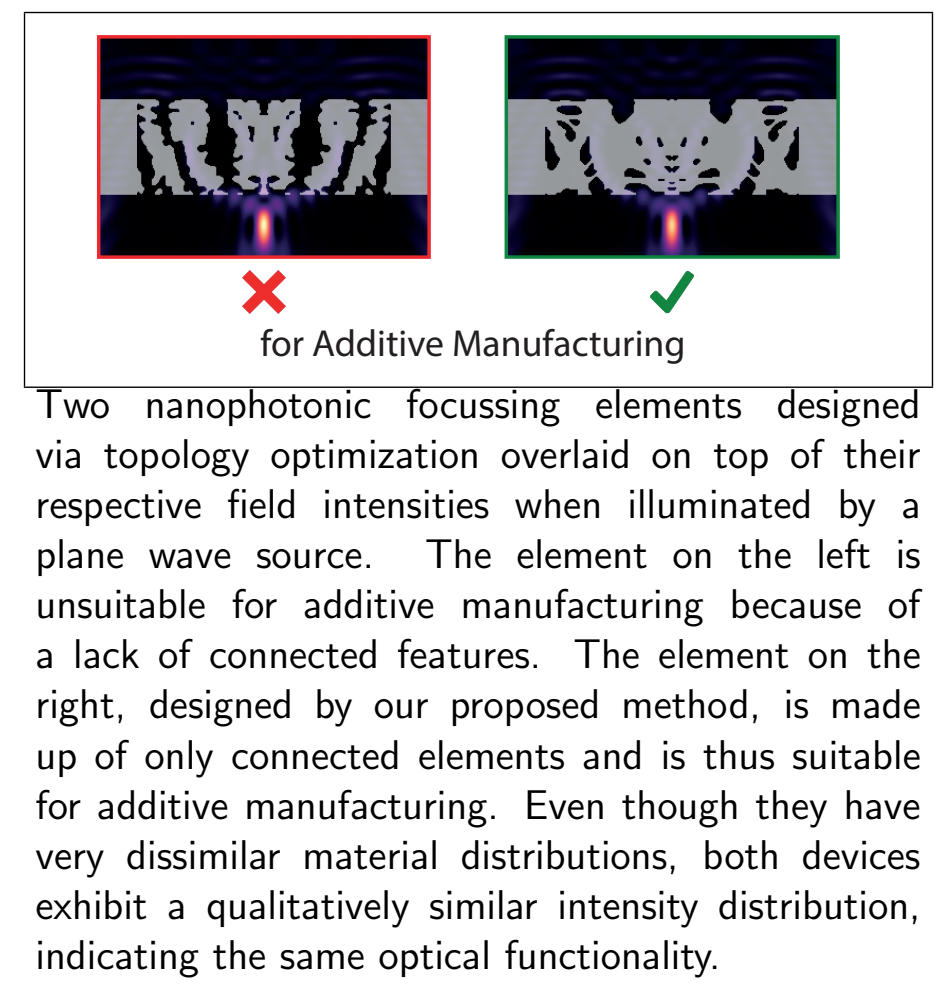

